\title{
Total Knee Arthroplasty After High Tibial Osteotomy
}

\section{No Differences Between Medial and Lateral Osteotomy Approaches}

\author{
Stephen Preston MDCM, James Howard MD, FRCSC, \\ Douglas Naudie MD, FRCSC, Lyndsay Somerville PhD, \\ James McAuley MD, FRCSC
}

Published online: 9 May 2013

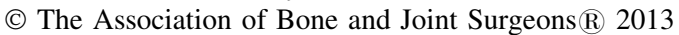

\begin{abstract}
Background High tibial osteotomy (HTO) has long been accepted as an effective treatment for unicompartmental osteoarthritis of the knee in young, active adults. For varus knees, the two most commonly performed valgus-producing HTOs are the lateral closing wedge and the medial opening wedge. Regardless of technique, some HTOs fail and are converted to TKA. To our knowledge, no studies have directly compared TKAs done after lateral closingwedge osteotomies to those done after medial openingwedge osteotomies.

Questions/purposes We compared pain- and functionrelated outcomes and survivorship of TKAs in patients who
\end{abstract}

The institution of the authors has received, during the study period, funding from Stryker Orthopaedics (Mahwah, NJ, USA), Smith \& Nephew Inc (Memphis, TN, USA), and DePuy Orthopaedics, Inc (Warsaw, IN, USA).

One of the authors certifies that he (JM) has received payments or benefits, during the study period, of less than USD 10,000 from DePuy. One of the authors certifies that he (DN) has received payments or benefits, during the study period, of less than USD 10,000 from Smith \& Nephew and Stryker. One of the authors certifies that he $(\mathrm{JH})$ has received payments or benefits, during the study period, of less than USD 10,000 USD from Smith \& Nephew, Stryker, and DePuy.

All ICMJE Conflict of Interest Forms for authors and Clinical Orthopaedics and Related Research editors and board members are on file with the publication and can be viewed on request. Each author certifies that his or her institution approved the human protocol for this investigation, that all investigations were conducted in conformity with ethical principles of research, and that informed consent for participation in the study was obtained.

S. Preston, J. Howard, D. Naudie, L. Somerville,

J. McAuley $(\square)$

London Health Sciences Center-University Hospital, B9-006,

London, Ontario N6A 5A5, Canada

e-mail: James.McAuley@lhsc.on.ca previously underwent medial opening-wedge and lateral closing-wedge HTOs.

Methods We reviewed our institutional database to identify all patients having undergone a HTO with subsequent conversion to TKA and identified 188 lateral closing-wedge HTOs and 77 medial opening-wedge HTOs. Mean followup was 88 months (range, 3-264 months) for the lateral closing-wedge HTO cohort and 59 months (range, 3-180 months) for the medial opening-wedge cohort. SF-12, WOMAC, and Knee Society Scores, as well as survivorship at 5 years, were compared between the two groups. Statistical analysis was completed using the independent-samples t-test.

Results No differences were found between groups in postoperative SF-12 scores $(\mathrm{p}=0.77$ for the mental component summary score and $\mathrm{p}=0.21$ for the physical component summary score), WOMAC scores $(\mathrm{p}=0.67)$, or Knee Society Scores $(\mathrm{p}=0.80)$. No difference was found in survivorship between groups at 5 years.

Conclusions Our results suggest there is no difference in functional outcomes or survivorship of TKA in patients having previously undergone medial opening-wedge and lateral closing-wedge HTOs.

Level of Evidence Level III, therapeutic study. See Instructions for Authors for a complete description of levels of evidence.

\section{Introduction}

Valgus-producing high tibial osteotomy (HTO) is an accepted treatment option for young, active patients with varus gonarthrosis. By changing the mechanical axis of the lower limb, the arthritic medial compartment of the knee is unloaded, providing patients with reliable pain relief. The 
two most common approaches to valgus-producing HTOs are the lateral closing wedge and the medial opening wedge. Traditionally, lateral closing-wedge HTOs were more common; however, in the late 1990s and early 2000s, medial opening-wedge HTOs became much more prevalent at our institution [2]. Advantages of this approach include greater control over degree of deformity correction, preservation of proximal tibial bone stock, less extensive soft tissue dissection, sparing of the proximal tibiofibular joint, lower reported incidence of compartment syndrome, and lower incidence of peroneal nerve injury [5]. Today, our center performs an average of 35 medial openingwedge HTOs per year [1].

A study from our institution by Naudie et al. [8] demonstrated excellent long-term survivorship of HTOs in carefully selected patients. Unfortunately, degenerative changes in the knee tend to progress despite initially successful realignment procedures and some HTOs are converted to TKA. However, given that the mechanical and anatomic axes of the tibia have been altered in these patients, performance of arthroplasty after HTO has been extensively reviewed in the literature $[1,3,4,6,7,9,10]$. The vast majority of these data deal with outcomes of TKA after conversion from lateral closingwedge HTOs. Although somewhat conflicting, recent data indicate lateral closing-wedge HTOs converted to TKA perform as well as primary TKAs $[4,10]$. Only one study exists comparing short-term functional outcomes of converted medial opening-wedge HTO to primary TKA. These results suggest inferior performance of arthroplasty after osteotomy at 2 years [1]. This is somewhat disconcerting given the volume of medial opening-wedge HTOs performed at our center with an estimated cumulative rate of conversion to TKA of $10 \%$ [1]. To our knowledge, no published data currently exist directly comparing functional outcomes of converted medial opening-wedge HTOs to converted lateral closing-wedge HTOs. Survivorship of TKA after conversion from HTO has also come into question in the literature. A study by Haslam et al. [4] suggested a trend toward poorer survivorship of converted lateral closing-wedge HTOs at 12 years when compared to primary TKA. However, no statistically significant difference was found. No data exist comparing survivorship of converted lateral closing-wedge HTOs to medial opening-wedge HTOs.

We therefore compared (1) pain- and function-related outcome scores and (2) survivorship of TKA between patients with TKA who were previously treated with either a lateral closing-wedge HTO or medial opening-wedge HTO.

\section{Patients and Methods}

Institutional ethics approval was obtained for a retrospective cohort study design to compare most recent functional outcomes (WOMAC score, Knee Society Score [KSS], and SF-12 score) and 5-year survivorship of patients having undergone TKA in the setting of previous lateral closingwedge or medial opening-wedge HTO at our institution. We then conducted a retrospective review of our institution's database to identify all patients having undergone an HTO that required subsequent conversion to TKA from 1996 to 2011. Only valgus-producing HTOs were eligible for inclusion in our study. Our study population was then divided into lateral closing-wedge and medial openingwedge cohorts. Electronic and physical chart review was carried out for all participants to assess clinical outcomes and survivorship of TKA.

Only patients with sufficient data sets were eligible for inclusion in the study. Patients were excluded for insufficient data if one of the outcome measures was absent from the database and physical chart. Review of the database revealed 294 patients who had undergone conversion of HTO to TKA. Two hundred fifteen patients had a converted lateral closing-wedge HTO, with the remaining 79 having a converted medial opening-wedge HTO. The choice between lateral closing-wedge HTO versus a medial opening-wedge HTO was made by the treating surgeon at the time of the index procedure. Twenty-seven patients $(12.5 \%)$ from the lateral closing-wedge cohort were eliminated due to insufficient data whereas only two $(2.6 \%)$ from the medial opening-wedge cohort were eliminated for this reason $(\mathrm{p}<0.001)$. There was also a statistically significant difference in the loss to followup between the two groups: $22 \%$ for the lateral closing-wedge cohort versus $10 \%$ in the medial opening-wedge cohort $(\mathrm{p}<0.001)$. Unsuccessful attempts were made to contact these patients for further followup. Nonetheless, their most recent data were included in the analysis. Thus, we were left with 188 patients in the lateral closing-wedge cohort and 77 in the medial opening-wedge cohort for data analysis (Fig. 1). Demographic analysis of the two cohorts revealed no difference in male to female ratio or average BMI. However, statistically significant differences were found for average age at conversion to TKA $(\mathrm{p}<0.001)$ and average length of followup $(\mathrm{p}<0.001)$ (Table 1). Mean followup was 88 months (range, 3-264 months) for the lateral closingwedge HTO cohort and 59 months (range, 3-180 months) for the medial opening-wedge cohort. Comparison of preoperative scores for functional measures (WOMAC score, KSS, and SF-12 score) showed no differences, indicating the two cohorts were similar in terms of the subjective and objective performance of their knees before conversion of HTO to TKA (Table 2).

Clinical outcomes were assessed using the WOMAC, KSS, and SF-12 questionnaires. The most recent scores available in the patients' charts were used for comparison. Patients at our institution are asked to fill out these 
Fig. 1 A flowchart demonstrates the distribution of patients between the medial lateral-closing and medial opening-wedge cohorts, mortality, and loss to followup.

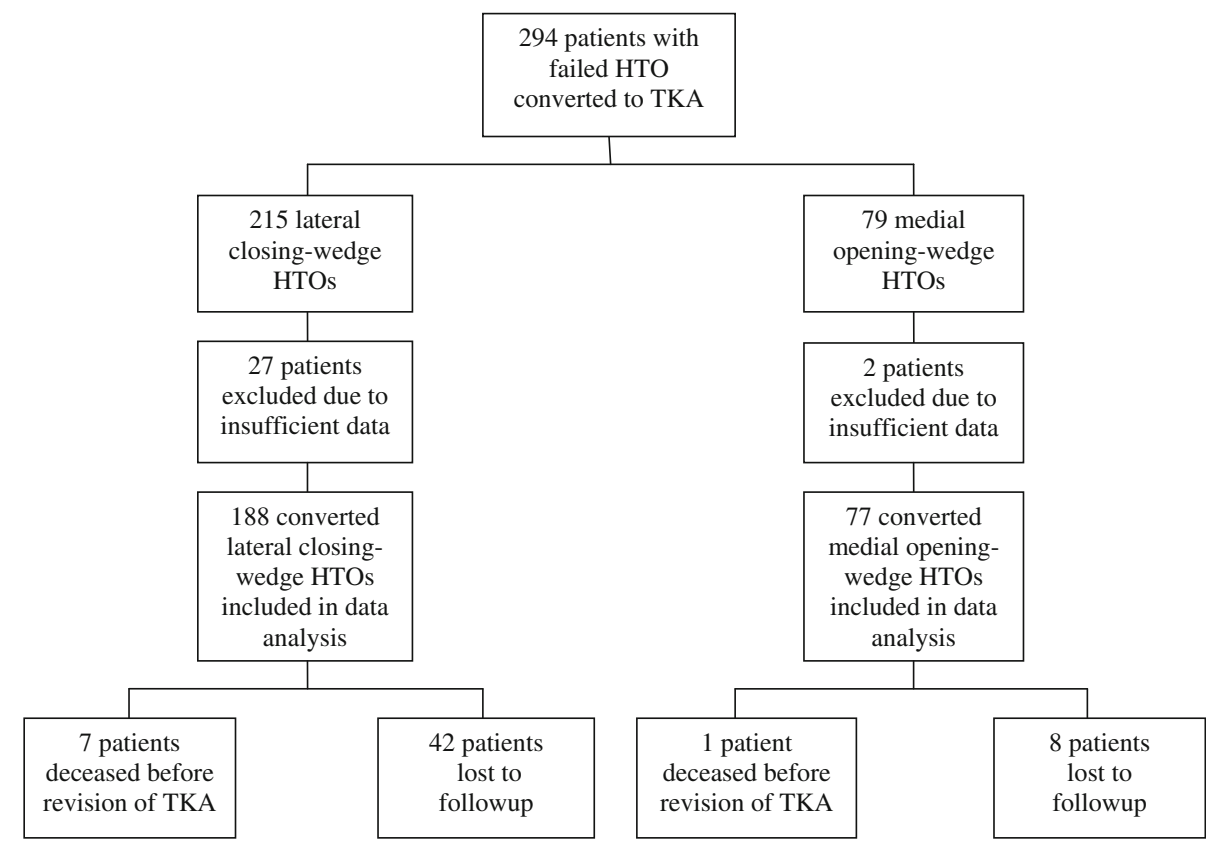

Table 1. Patient demographics

\begin{tabular}{llll}
\hline Variable & $\begin{array}{l}\text { Lateral closing- } \\
\text { wedge HTO } \\
\text { cohort }\end{array}$ & $\begin{array}{l}\text { Medial opening- } \\
\text { wedge HTO } \\
\text { cohort }\end{array}$ & p value \\
\hline $\begin{array}{l}\text { Number of } \\
\text { patients }\end{array}$ & 188 & 77 & \\
$\begin{array}{l}\text { Mean age at } \\
\text { conversion } \\
\text { (years) }\end{array}$ & 61.4 & 57.9 & $<0.001$ \\
$\begin{array}{l}\text { Mean followup } \\
\text { (months) }\end{array}$ & 88 & 59 & $<0.001$ \\
$\begin{array}{l}\text { Mean BMI } \\
\text { Females (\%) }\end{array}$ & 32.7 & 32.2 & 0.52 \\
Males (\%) & 56.9 & 39.5 & 0.59 \\
\hline
\end{tabular}

HTO = high tibial osteotomy.

assessment tools at all followup clinic visits (at 3 months, 6 months, 1 year, 2 years, and every 2 years thereafter). The physician portion of the KSS was completed by the attending surgeon, clinical fellow, resident, or research nurse who assessed the patients during their followup visits.

Student's independent t-tests were used to compare the mean functional scores for each variable (WOMAC score, KSS, and SF-12 score) between our two study cohorts, with $\mathrm{p}$ values of less than 0.05 considered statistically significant. Survivorship of TKA was calculated using the length of time elapsed from conversion of the HTO to revision of the TKA. Mean survivorship was plotted for the two cohorts on Kaplan-Meyer survivorship curves with $95 \%$ CIs. Curves included data points until 5-year followup and were performed for both all-cause and aseptic revisions.
Table 2. Preoperative functional scores

\begin{tabular}{llll}
\hline Scoring system & $\begin{array}{l}\text { Lateral closing- } \\
\text { wedge HTO } \\
\text { cohort }\end{array}$ & $\begin{array}{l}\text { Medial opening- } \\
\text { wedge HTO } \\
\text { cohort }\end{array}$ & p value \\
\hline $\begin{array}{l}\text { Mean SF-12 score (points) } \\
\text { Mental }\end{array} \quad$ 51.56 & 51.84 & 0.87 \\
component & & & \\
summary & 30.50 & 30.27 & 0.86 \\
Physical & & & \\
component & & & \\
summary & 42.92 & 43.21 & 0.92 \\
Mean WOMAC score (points) & 38.84 & 0.88 \\
Pain & 38.35 & 46.69 & 0.39 \\
Stiffness & 44.30 & 43.58 & 0.67 \\
Function & 42.57 & & \\
Total & 14.79 & 48.45 & 0.64 \\
Mean Knee Society Score (points) & & 0.86 \\
Pain & 48.56 & 40.45 & 0.65 \\
Function & 41.51 & 89.20 & 0.83 \\
Knee & 90.02 & & \\
Total & & & \\
\hline
\end{tabular}

$\mathrm{HTO}=$ high tibial osteotomy.

\section{Results}

Comparison of the most recent functional outcome scores after conversion to TKA revealed no differences between the lateral closing-wedge and medial opening-wedge cohorts. SF-12 scores were not different for either the mental component summary score $(p=0.77)$ or the physical component summary score $(\mathrm{p}=0.21)$ (Fig. 2). Comparison of WOMAC scores revealed no differences for 
pain, stiffness, function, and total scores $(\mathrm{p}=0.71$, $\mathrm{p}=0.36, \mathrm{p}=0.86$, and $\mathrm{p}=0.67$, respectively) (Fig. 3). There were no differences in ROM between groups (Fig. 4). Finally, no differences were found between groups in the KSS (pain, function, knee, and total scores: $\mathrm{p}=0.45$, $\mathrm{p}=0.1, \mathrm{p}=0.95$, and $\mathrm{p}=0.18$, respectively) (Fig. 5).

For all-cause revisions, 5-year survivorship for TKAs in the lateral closing-wedge cohort was $95 \% \quad(95 \% \mathrm{CI}$ : 94\%-97\%) compared to $95 \%$ (95\% CI: 93\%-98\%) for the medial opening-wedge cohort (Fig. 6). For aseptic revisions, average 5-year survivorship for the lateral closingwedge cohort was $97 \%$ (95\% CI: $96 \%-98 \%$ ) compared to 97\% (95\% CI: 94\%-99\%) for the medial opening-wedge cohort (Fig. 7). No significant differences were found for any of the above-mentioned comparisons.

\section{Discussion}

Valgus-producing HTO has long been considered an accepted treatment option for young, active patients suffering from varus gonarthrosis. The medial opening-wedge HTO has gradually taken the place of the lateral closingwedge HTO as the osteotomy of choice at our center [2]. Some HTOs fail from progression of the arthritic process and are converted to TKAs. To our knowledge, no studies have compared TKAs done after medial opening-wedge osteotomies to TKAs done after lateral closing-wedge osteotomies. We therefore compared pain- and functionrelated outcomes and survivorship of TKAs in patients having previously undergone medial opening-wedge and lateral closing-wedge HTOs.

The most important limitation to our study is the potential selection bias introduced at the time of the index surgery (valgus-producing HTO). The choice of lateral

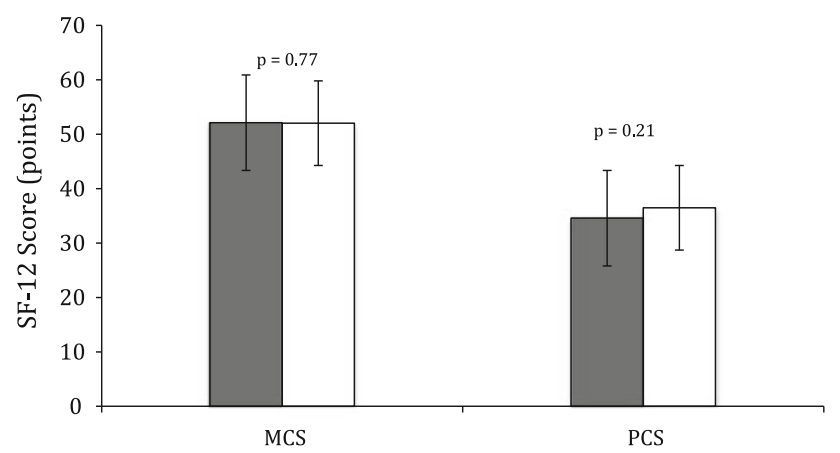

Fig. 2 A graph shows latest SF-12 scores in patients converted to TKA from lateral closing-wedge and medial opening-wedge HTOs. Dark gray and white bars represent the lateral closing-wedge and medial opening-wedge cohorts, respectively. SF-12 scores were not different between groups for either the mental component summary score $(\mathrm{MCS})(\mathrm{p}=0.77)$ or the physical component summary score (PCS) $(\mathrm{p}=0.21)$. closing-wedge versus medial opening-wedge HTO was made by the treating surgeon based on personal experience and comfort level. Many of the HTOs included in this study were performed during a time period where a shift from

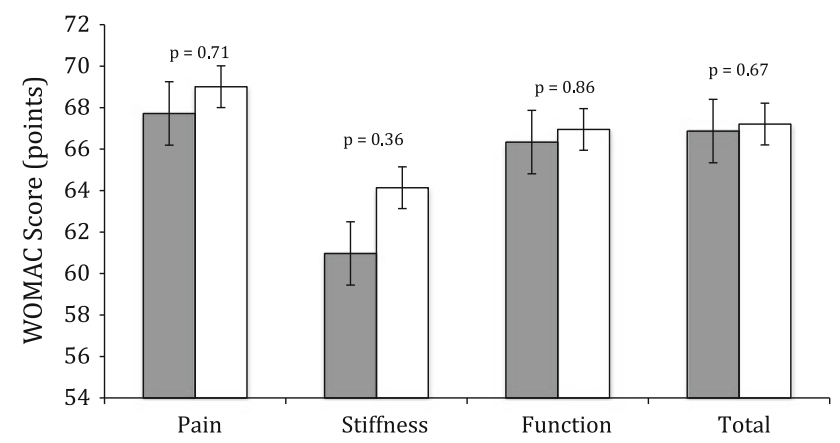

Fig. 3 A graph shows latest WOMAC scores in patients converted to TKA from lateral closing-wedge and medial opening-wedge HTOs. Dark gray and white bars represent the lateral closing-wedge and medial opening-wedge cohorts, respectively. WOMAC scores revealed no differences between groups for pain, stiffness, function, and total scores $(\mathrm{p}=0.71, \mathrm{p}=0.36, \mathrm{p}=0.86$, and $\mathrm{p}=0.67$, respectively).

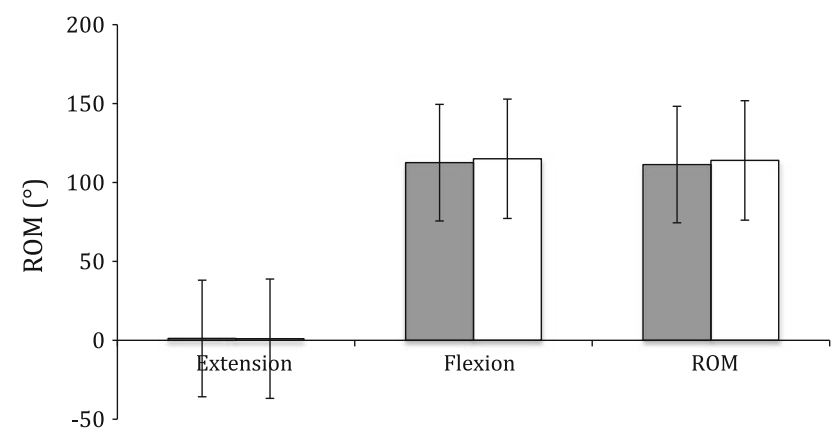

Fig. 4 A graph shows latest knee ROM in patients converted to TKA from lateral closing-wedge and medial opening-wedge HTOs. Dark gray and white bars represent the lateral closing-wedge and medial opening-wedge cohorts, respectively. No difference between groups was found for this outcome measure.

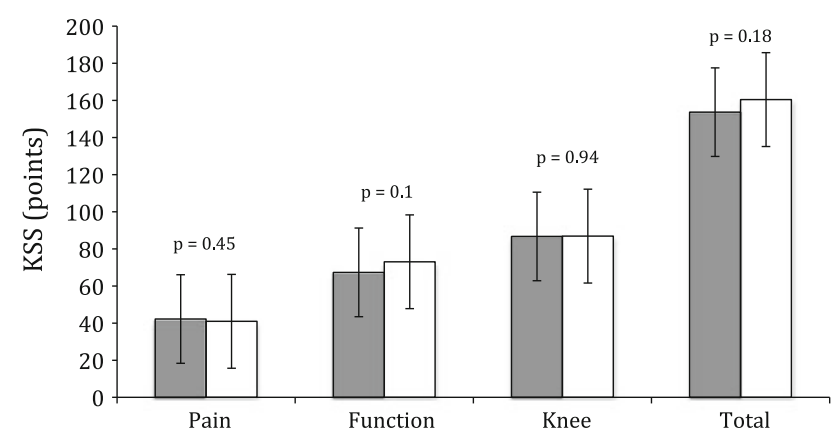

Fig. 5 A graph shows latest KSSs in patients converted to TKA from lateral closing-wedge and medial opening-wedge HTOs. Dark gray and white bars represent the lateral closing-wedge and medial opening-wedge cohorts, respectively. KSSs revealed no differences between groups for pain, function, knee, and total scores $(\mathrm{p}=0.45$, $\mathrm{p}=0.1, \mathrm{p}=0.95$, and $\mathrm{p}=0.18$, respectively). 


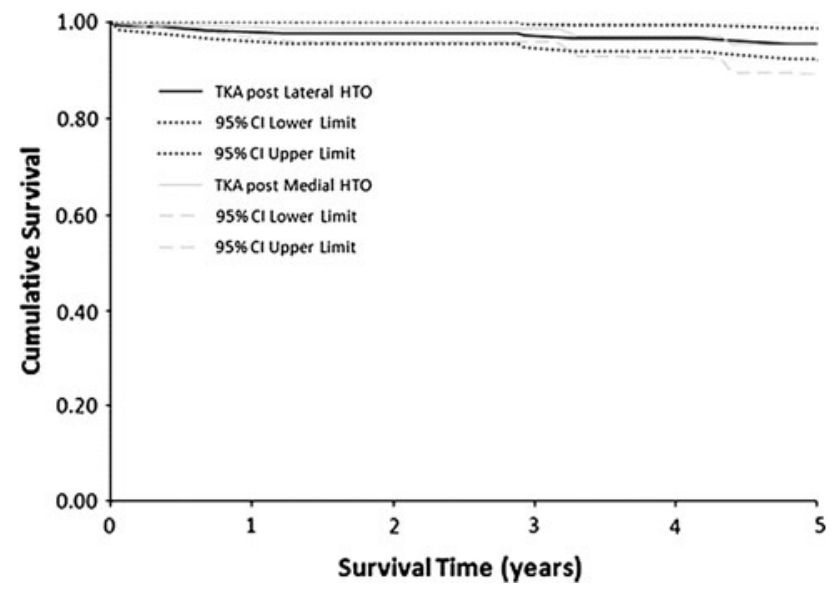

Fig. 6 Kaplan-Meier survivorship curves with revision for any reason as the end point are shown in patients converted to TKA from lateral closing-wedge and medial opening-wedge HTOs. Average 5-year survival rates were 95\% (95\% CI: 94\%-97\%) for the lateral closing-wedge cohort and 95\% (95\% CI: 93\%-98\%) for the medial opening-wedge cohort.

lateral closing-wedge HTOs to medial opening-wedge HTOs was taking place. Thus, surgeons had less experience with the medial opening-wedge HTO. Lack of experience could have made preservation of slope and appropriate tibial rotation more difficult, thereby negatively affecting performance and survival of subsequent TKA in this cohort. In addition, the differences in patient exclusion due to missing data and loss to followup were substantial between the lateral closing-wedge and medial openingwedge cohorts. Unfortunately, many lateral closing-wedge HTOs were converted to TKA during a time when data were less rigorously collected during clinical followup at our institution. A shift toward medial opening-wedge HTOs and their subsequent conversion to TKA coincided with improvements in data collection. This is clearly reflected in the number of incomplete data sets for the lateral closing-wedge cohort, the effect of which on our results cannot be determined at this time. The vast majority of patients who were lost to followup ceased to attend clinic visits later than 10 years after conversion to TKA. An Internet search of obituaries and review of our hospital records were undertaken to ensure that these patients had not expired. Nevertheless, the most recent complete data sets for these patients were included in our data analysis. Finally, the lateral closing-wedge and medial openingwedge cohorts were unmatched at the time of data analysis. The lateral closing-wedge HTO is a much older procedure than its medial opening-wedge counterpart. Thus, lateral closing-wedge HTOs have had more time to fail and require conversion to TKA, resulting in much higher numbers in this cohort. Given the fact that our center currently performs medial opening-wedge HTOs almost exclusively, more time would be required to obtain

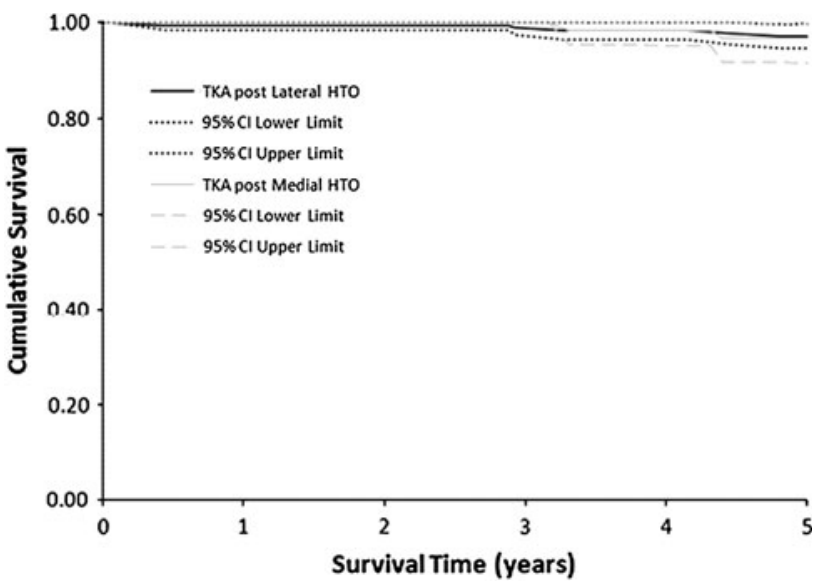

Fig. 7 Kaplan-Meier survivorship curves with aseptic revisions only as the end point are shown in patients converted to TKA from lateral closing-wedge and medial opening-wedge HTOs. Average 5-year survival rates were $97 \%$ (95\% CI: $96 \%-98 \%$ ) for the lateral closingwedge cohort and 96\% (95\% CI: 94\%-99\%) for the medial openingwedge cohort.

matched cohorts without affecting patient numbers. This would also allow us to extend our survivorship analysis beyond 5 years for the medial opening-wedge cohort.

Functional outcomes as measured by the most recent SF-12 score, WOMAC score, ROM, and KSS were not different between the lateral closing-wedge and medial opening-wedge cohorts. To date, no such comparisons exist in published literature. Existing data comparing functional outcomes and survivorship of converted lateral closingwedge HTOs to primary TKAs are somewhat mixed. Karabatsos et al. [6] demonstrated that TKA in the setting of a previous HTO is more technically demanding than primary TKA. These authors demonstrated a trend toward inferior functional scores in converted lateral closingwedge HTOs at 5 years, although no statistically significant differences were found. In a similar comparison, Nizard et al. [9] found a significant difference only in pain scores in patients after conversion of lateral closing-wedge HTO compared to primary TKA. Haslam et al. [4] demonstrated significant differences in the number of poor results, knee flexion, and failures at 12.6-year followup for patients with converted lateral closing-wedge HTOs. However, they found no significant differences in overall Hospital for Special Surgery scores when these patients were compared to a matched cohort of primary TKAs. In addition, a systematic review of functional outcomes of converted lateral closing-wedge HTOs compared to those of primary TKA revealed no differences between these two groups [10]. Conversely, the only study comparing the results of converted medial opening-wedge HTOs to those of primary TKA suggested converted HTOs performed worse at 2-year followup [1]. However, that study included only 34 knees at the time of data analysis. 
Survivorship rates of TKAs in our lateral closing-wedge and medial opening-wedge cohorts were also similar for all-cause and aseptic revisions. To our knowledge, no similar comparisons exist in the literature.

We found functional outcomes for TKA after conversion from lateral closing-wedge HTO to be similar to those after conversion from medial opening-wedge HTO. Survivorship at 5 years also was similar between these two cohorts. To our knowledge, this is the first study to directly compare the results in these two groups of patients. Nevertheless, further followup is required to obtain an adequate sample size to draw meaningful conclusions regarding long-term survivorship of TKA after conversion from medial opening-wedge HTO.

\section{References}

1. Erak S, Naudie D, MacDonald SJ, McCalden RW, Rorabeck CH, Bourne RB. Total knee arthroplasty following medial opening wedge high tibial osteotomy: technical issues early clinical radiological results. Knee. 2011;18:499-504.
2. Fowler PJ, Tan JL, Brown GA. Medial opening wedge osteotomy: how I do it. Oper Tech Sports Med. 2000;8:32-38.

3. Haddad FS, Bentley G. Total knee arthroplasty after high tibial osteotomy: a medium-term review. J Arthroplasty. 2000;15: 597-603.

4. Haslam P, Armstrong M, Geutjens G, Wilton TJ. Total knee arthroplasty after failed high tibial osteotomy: long-term followup of matched groups. J Arthroplasty. 2007;22:245-250.

5. Hooper G, Leslie H, Burn J, Schouten R, Beci I. Oblique upper tibial opening wedge osteotomy for genu varum. Oper Orthop Traumatol. 2005;17:662-673.

6. Karabatsos B, Mahomed NN, Maistrelli GL. Functional outcome of total knee arthroplasty after high tibial osteotomy. Can J Surg. 2002;45:116-119.

7. Meding JB, Keating EM, Ritter MA, Faris PM. Total knee arthroplasty after high tibial osteotomy. Clin Orthop Relat Res. 2000;375:175-184.

8. Naudie D, Bourne RB, Rorabeck CH, Bourne TJ. The Insall Award. Survivorship of the high tibial valgus osteotomy: a 10- to 22-year followup study. Clin Orthop Relat Res. 1999;367:18-27.

9. Nizard R, Cardinne L, Bizot P, Witvoet J. Total knee replacement after failed tibial osteotomy: results of a matched pair study. J Arthroplasty. 1998;13:847-853.

10. Van Raaij TM, Reijman M, Furlan AD, Verhaar JA. Total knee arthroplasty after high tibial osteotomy: a systematic review. BMC Musculoskelet Disord. 2009;10:1186-1195. 